\title{
Visible Light Flow Reactor Packed with Porous Carbon Nitride for Aerobic Substrate Oxidations
}

Mark A. Bajada, ${ }^{a}$ Arjun Vijeta, ${ }^{a}$ Aleksandr Savateev,${ }^{\mathrm{b}}$ Guigang Zhang, ${ }^{\mathrm{b}}$ Duncan Howe, ${ }^{\mathrm{a}}$ and Erwin Reisner*a

a Department of Chemistry, University of Cambridge, Lensfield Road, Cambridge CB2 $1 E W(U K)$

E-mail: reisner@ch.cam.ac.uk

Homepage: http://www-reisner.ch.cam.ac.uk

${ }^{b}$ Department of Colloid Chemistry, Max Planck Institute of Colloids and Interfaces, 14476 Potsdam (Germany) 


\section{Abstract}

A triphasic photocatalytic reactor employing a mesoporous carbon nitride photocatalyst and aerobic $\mathrm{O}_{2}$ was assembled to operate under continuous flow conditions. This reactor design allows for facile downstream processing and reusability in multiple flow cycles. The selective aerobic oxidation of alcohols and amines was chosen to demonstrate the applicability and performance advantage of this flow approach compared to conventional batch photochemistry. This precious-metal-free photocatalytic flow system operates under benign reaction conditions (visible light, low pressure, and mild temperature) and will stimulate the exploration of other oxidative reactions in a sustainable, scalable, and affordable manner.

Keywords: photocatalysis, flow chemistry, aerobic oxidation, carbon nitride, green chemistry

\section{Introduction}

Continuous flow chemistry has grown tremendously in recent years due to its advantages over conventional batch reactors, particularly for photocatalytic processes. ${ }^{1-5}$ Batch photoreactors tend to be comprised of a simple tube or flask, and hence suffer from a lack of design specificity towards light driven transformations. Alternatively, flow devices can be fabricated to have a high surface area-to-volume ( $S A$-to- $V$ ) ratio, guaranteeing extensive transmission of light throughout the reaction mixture even at high chromophore concentrations. ${ }^{6}$ Furthermore, this characteristic also ensures efficient thermal transfer, reducing the risk of runaway reactions from exothermic pathways. ${ }^{7,8}$ The continuous operation also allows for accurate control of residence and irradiation time via the flow rate. ${ }^{9}$ Photocatalyst immobilization within the reactor can assist with downstream processing of reaction mixtures as it avoids catalyst separation and thereby facilitates reusability and scalability. ${ }^{10}$

Progress in the field of photoredoxcatalysis has mostly been confined to homogeneous systems, involving costly $\mathrm{Ru}$ and $\mathrm{Ir}$ complexes, ${ }^{11}$ and tailor-made organic chromophores. ${ }^{12}$ Graphitic carbon nitride, a polymeric, easily synthesizable, robust, metal-free material composed of tri-s-triazine units (Figure S1), ${ }^{13}$ is one of the most promising heterogeneous photocatalysts, with applications in visible light 
mediated organic transformations, ${ }^{14,15}$ and early-stage development in flow photochemistry. ${ }^{16,17}$

Two main approaches for carbon nitride-based flow photochemistry have been adopted to date: (i) featuring a stationary carbon nitride phase within a packed column approach, ${ }^{16}$ and (ii) using a suspension in-flow, that is divided into smaller sub-units by means of an inert gas spacer (termed serial micro-batch reactors, SMBR). ${ }^{17}$ The former approach allows for the effective immobilization of the carbon nitride material, and the overall setup can therefore easily be re-used with minimal effort to recover the photocatalyst. However, this configuration was limited to liquid-only flow applications, and not for triphasic (gas-liquid-solid) flow chemistry. The innovative design of the SMBR made use of toroidal vortices in the segmented flow pattern to produce 'wellstirred' micro-suspensions containing the carbon nitride photocatalyst. This internal mixing phenomenon was therefore responsible for homogenizing the mixture and resulted in the formation of uniformly irradiated suspensions in the flow sequence. However, the separation strategy for product isolation, and recovery of the catalyst and ionic liquid was demanding (featuring filtration, liquid extraction, and solid extraction stages), and hence offered some additional complexity to the reaction protocol in comparison to the packed column design. The SMBR employed inert gas $\left(\mathrm{N}_{2}\right)$ to generate the flow pattern, and hence the study precluded the use of a reactive atmosphere.

The demand from industry for aerobic oxidation catalysts is growing. ${ }^{18} \mathrm{O}_{2}$ can be considered as an ideal oxidant, as it is readily abundant and usually generates water as a benign by-product. This is especially advantageous for the production of active pharmaceutical ingredients, where impurities are strongly regulated. ${ }^{19} \mathrm{~A}$ review of large-scale oxidation processes concluded that $\mathrm{O}_{2}$ gas has the advantage of being a cost-effective and readily available oxidant, but the safety issues surrounding its use (mixtures of $\mathrm{O}_{2}$ and organic solvents can potentially generate highly flammable mixtures) make it one of the least frequently used reagents. ${ }^{20}$ The recent revival in the development of catalytic systems for aerobic substrate oxidations is testimony to the commitment from industry to pursue the application of aerobic $\mathrm{O}_{2}$. However, such systems are either confined to a small scale, ${ }^{21}$ feature catalysts containing precious metals, ${ }^{22}$ make use of a homogeneous photocatalyst that hinders downstream processing and separation, ${ }^{23,24}$ or are unsuitable for a continuous flow platform. ${ }^{25}$ 
Conducting aerobic oxidations in-flow with a carbon nitride material is therefore an attractive option due to the attributes of this photocatalyst, and the potential to explore this chemistry at a more commercial level. The packed column design offers both a large interfacial area and simulates high local catalyst loading, ${ }^{7,26,27}$ and is in fact more applicable when heterogeneous catalysis is needed for continuous, multiphase (gas-liquid-solid) chemical transformations, such as catalytic hydrogenation reactions. ${ }^{28,29}$

Here we present a triphasic, packed column photoreactor (PCP) system for the continuous flow oxidative photochemistry of alcohols and amines during visible light irradiation, in which a mesoporous, graphitic carbon nitride material $\left(\mathbf{m p g}-\mathbf{C N}_{\mathbf{x}}\right)$ is incorporated as the stationary photocatalyst, and aerobic $\mathrm{O}_{2}$ is used as the terminal electron acceptor and oxidant (Figure 1, Scheme 1). In addition, we demonstrate that this chemistry can be accomplished using more benign conditions compared to previously reported $\mathbf{m p g}-\mathbf{C N}_{\mathbf{x}}$-based batch photoreactors, which typically require temperatures and $\mathrm{O}_{2}$ pressures in the range of $80-100{ }^{\circ} \mathrm{C}$ and $5-8$ bar, respectively. ${ }^{30,31}$ The previously proposed photocatalytic mechanism for this reaction suggests that photoactivated $\mathbf{m p g}-\mathbf{C N}_{\mathbf{x}}$ oxidizes the benzyl alcohol/amine to the corresponding aldehyde/imine and reduces $\mathrm{O}_{2}$ to hydrogen peroxide and water (Figure S1). ${ }^{30}$ Aldehydes and imines are commonly used synthetic building blocks for the pharmaceutical industry. ${ }^{31}$

\section{Results and Discussion}

A flow setup was constructed to generate a stable gas-liquid segmented (slug) flow pattern (Figure 1). This pattern formed downstream of a 4-port 3-way valve, where the substrate and air fronts meet and mix. The resultant laminar flow feeds into the PCP, which was comprised of a flexible, transparent fluorinated ethylene propylene (FEP) tube $(I=75 \mathrm{~cm}$, i.d. $=2 \mathrm{~mm})$, which was packed with the carbon nitride material (3.5 wt.\% in a glass bead mixture; see Supporting Information for more details). The PCP was coiled around a reflective support, and then irradiated in a $360^{\circ}$ fashion with a blue LED setup ( $14.4 \mathrm{~W}$ at $\lambda=470 \mathrm{~nm}$ ). Typically, $2.5 \mathrm{~mL}$ of the substrate solution (concentration in the range of $50-100 \mathrm{mM}$ ) was first injected into the reactor, followed by $2.5 \mathrm{~mL}$ of pure solvent. The residence time of the starting material, $\tau$, within the 
photoactive zone was varied by changing the flow rate as the reactor volume was kept fixed at $\sim 1 \mathrm{~mL}$ (see Supporting Information, Eq. S2).

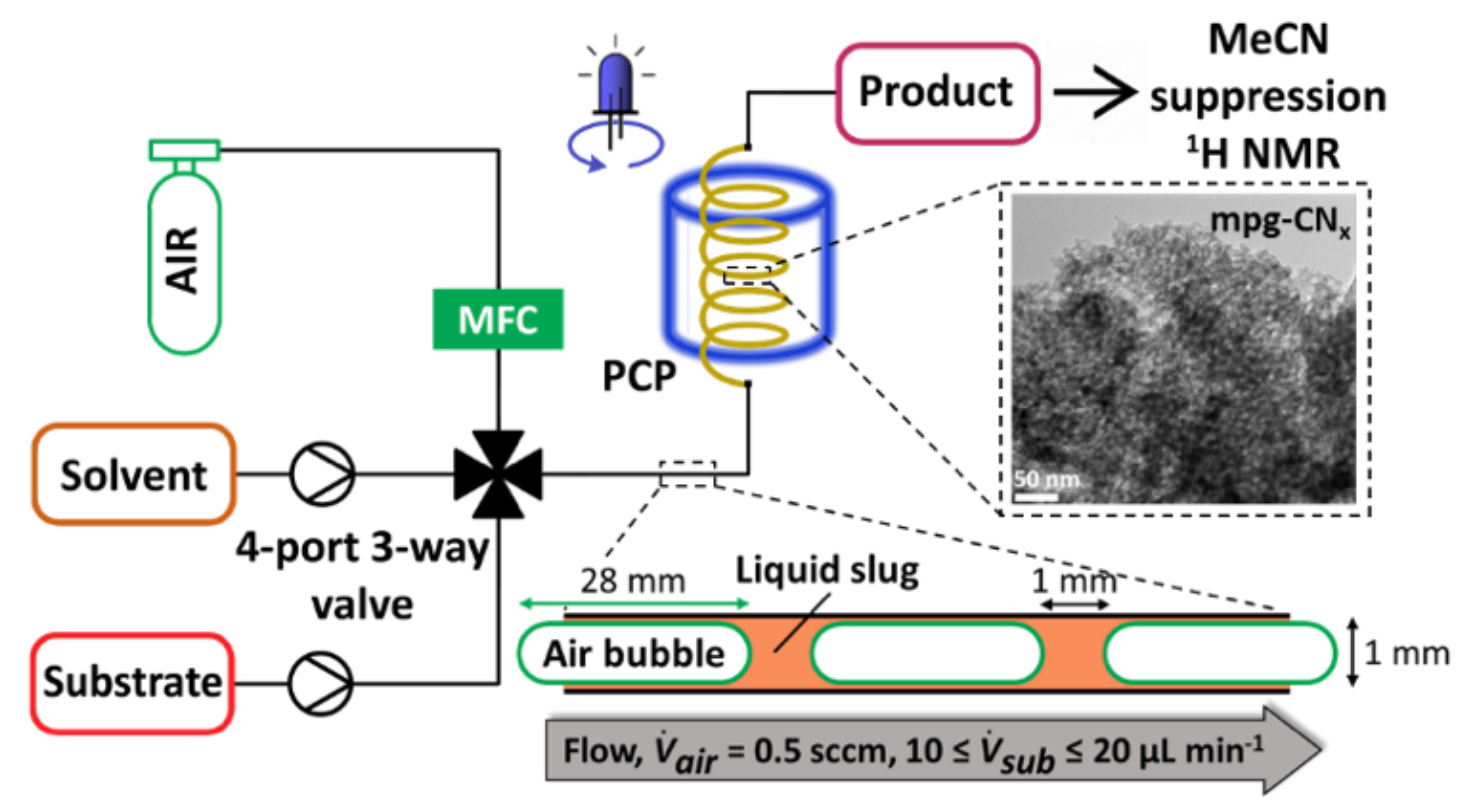

Figure 1. Schematic diagram of the triphasic flow photoreactor incorporating $\mathbf{m p g}-\mathbf{C N}_{\mathbf{x}}$ within the PCP. Irradiation is carried out via $360^{\circ}$ blue LEDs (14.4 W at $470 \mathrm{~nm}$ ) (MFC: mass flow controller). TEM image highlighting the mesoporous morphology of the $\mathbf{m p g}-\mathbf{C N}_{\mathbf{x}}$ material and illustration of the gasliquid flow injected into the PCP is also shown.

We observed that the morphology of the carbon nitride greatly impacts the flow performance of the PCP. In preliminary tests, both $\mathbf{m p g}-\mathbf{C N}_{\mathbf{x}}$ and conventional bulk carbon nitride $\left(\mathbf{b}-\mathbf{C N}_{\mathbf{x}}\right)$ were individually incorporated within the PCP. Transmission electron microscopy (TEM) images show the nonporous, flat morphology of $\mathbf{b}-\mathbf{C N}_{\mathbf{x}}$ (Figure S2a), which is in stark contrast to the mesoporous network of $\mathbf{m p g}-\mathbf{C N}_{\mathbf{x}}$ (Figure 1, Figure S2b). This morphological difference is also corroborated by $\mathrm{N}_{2}$ sorption measurements (Figure S3a), which demonstrate the large BrunauerEmmett-Teller (BET) surface area of $\mathbf{m p g}-\mathbf{C N}_{\mathbf{x}}\left(187 \mathrm{~m}^{2} \mathbf{g}^{-1}\right)$ in comparison to $\mathbf{b}-\mathbf{C N}_{\mathbf{x}}$ $\left(5 \mathrm{~m}^{2} \mathrm{~g}^{-1}\right)$. The pore size distribution gives an average pore size of $5.5 \mathrm{~nm}$ for $\mathbf{m p g}$ $\mathrm{CN}_{\mathbf{x}}$ (Figure S3b).

The $\mathbf{b}-\mathbf{C N}_{\mathbf{x}}$ PCP could not support the movement of a gas-liquid flow, presumably due to the agglomeration and clustering of the flat $\mathbf{b}-\mathbf{C N}_{\mathbf{x}}$ particles, which resulted in a high back-pressure $(\sim 1.5 \mathrm{MPa})$ during operation. On the other hand, the porous framework of $\mathbf{m p g}-\mathbf{C N}_{\mathbf{x}}$ allowed for facile gas-liquid permeation, making it suitable for flow applications. The absence of any critical back-pressure issues permitted the use of small diameter reactors, satisfying the high $S A$-to- $V$ constraint 
imposed for photoinduced reactions. The high $S A$-to- $V$ ratio is necessary in order to minimize the amount of photocatalyst (and hence reactive sites) within the central regions of the reactor which, as governed by the Beer-Lambert-Bouguer law, would experience a diminished photon flux. The larger surface area of $\mathbf{m p g}-\mathbf{C N}_{\mathbf{x}}$ compared to $\mathbf{b}-\mathbf{C N}_{\mathbf{x}}$ gives the additional benefit of improved charge separation and interfacial electron transfer. ${ }^{32}$

(a)

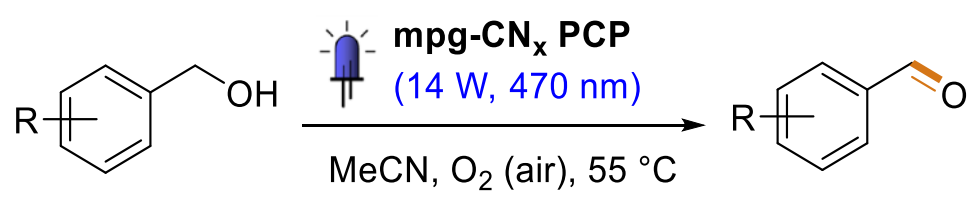

(b)

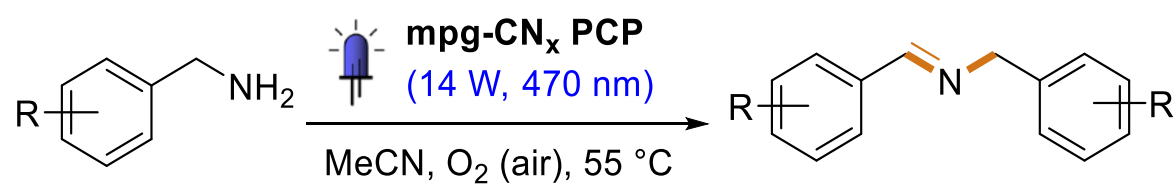

Scheme 1. (a) Alcohol and (b) amine oxidation reactions studied under continuous flow conditions.

The photoreactor assembly allowed for substrate and solvent injection, and the air feed was continuously switched on to ensure the formation of a gas-liquid flow, and to constantly supply aerobic $\mathrm{O}_{2}$ to the reactive centers. Acetonitrile $(\mathrm{MeCN})$ was the most effective solvent (Figure S4), and the heat generated from the LED setup $\left(\sim 55^{\circ} \mathrm{C}\right)$ increased the overall conversion yield. To facilitate downstream processing, an MeCN suppression pulse program for ${ }^{1} \mathrm{H}$ NMR analysis was designed and implemented, to allow for direct sampling and measurement of the crude mixture without the need for reaction treatment (see Supporting Information for more details).

The system was initially studied using the photocatalyzed aerobic oxidation of benzylic alcohols ${ }^{30}$ with 1 -naphthalenemethanol selected as the model compound (Scheme 1a, Figure 2). The overall starting material conversion (Conv), into the resultant aldehyde and carboxylic acid, and product selectivity (Sel) to the corresponding aldehyde, was controlled by varying the residence time in the PCP. The overall Conv approaches $100 \%$ at $\tau>90 \mathrm{~min}$, and the formation of the 'overoxidized' product (carboxylic acid) only becomes appreciable at $\tau>75 \mathrm{~min}$ (Figure 2). The significance of this result is that a lower value of $\tau$ could be employed to ensure high selectivity for the aldehyde; the starting material can then be separated from the reaction mixture in an in-line manner at the PCP exit and recycled back into the photoreactor setup. 
The fit applied to the Conv dataset in Figure 2 is of the form: $1-e^{-k_{P C P} \tau}$, where $k_{P C P}$ represents a form of 'rate constant' (units: $\min ^{-1}$ ) for the triphasic oxidation reaction within the PCP. KPCP is therefore expected to vary with both an alteration in the reactor parameters (such as catalyst loading, and diameter of the packed column, amongst others), and a change in substrate. Effectively, it can be used as a design guide to compare the performance of different reactor configurations (in the presence of the same substrate), or to compare the reactivity of different substrates within the same PCP design.

The inset in Figure 2 highlights the recyclability of the photocatalytic system, which maintains a high level of activity for multiple flow cycles. To compile this plot, a 1naphthalenemethanol solution in $\mathrm{MeCN}$ was injected into reactor $(2.5 \mathrm{~mL}, 100 \mathrm{mM})$, followed by $2.5 \mathrm{~mL}$ of pure MeCN. This procedure was repeated for a total of 25 times, and the Conv metric was computed after each individual flow reaction. Relative to the initial run, there was only an approximate $10 \%$ drop in activity by the $25^{\text {th }}$ flow experiment, most likely due to the sedimentation (and possible aggregation) of the mpg-CN $\mathbf{N}_{\mathbf{x}}$ particles within the packing mixture of the PCP (Figure S5), which would effectively lead to a reduction in the active surface area of the photocatalyst.

The benzylic oxidation reaction (Scheme 1a) was compared under both batch and flow conditions and the performance was assessed by determining a number of metrics, namely the Conv, Sel, and product-substrate recovery, with the latter henceforth denoted as the mass balance (M.B.). The $\boldsymbol{M}$. B. metric therefore provides a means to evaluate the moles of species exiting the reactor, relative to the amount injected into the system (see Supporting Information, Eq. S5). For the batch photoreactor, the reaction time was set to $90 \mathrm{~min}$ and the optimal $\mathbf{m p g}-\mathbf{C N}_{\mathbf{x}}$ loading was established (Figure S6). A wide range of para-substituted benzyl alcohols were studied, and the PCP setup displayed a substantial improvement in Conv compared to its batch counterpart (Table 1). The enhancement of the flow setup compared to batch stems from a higher local loading of the photocatalyst in the light-responsive zone; by directing more of the substrate over the catalyst, and constantly replenishing the $\mathrm{O}_{2}$ at the reactive sites (by means of the constant airflow), a higher activity can be achieved for a given reaction. The reaction time in the batch photoreactor had to be increased to $4 \mathrm{~h}$ in order to obtain a Conv of similar magnitude to that obtained under continuous flow conditions with $\tau$ set to $90 \mathrm{~min}$ (see Table 1, entry 1.i and 1, 
respectively). As in the case of the PCP, carboxylic acid formation in-batch became more appreciable for the higher Conv value. However, a better Sel for the aldehyde product was obtained for the flow photoreactor (93\% relative to $90 \%$ in-batch, Table 1 entry 1 and 1.i, respectively). This supports another general advantage of flow over batch photoreactors, in which the reaction mixture can be more easily removed from the photoactive zone, to minimize or lessen the formation of side-products resulting from secondary reactions or decomposition pathways ${ }^{33}$ (in this case, overoxidation of the aldehyde compound).

The external quantum efficiency (EQE) in-batch and in-flow was calculated to be $1.5 \%$ and $1.6 \%$ at $\lambda=470 \mathrm{~nm}$, respectively (see Supporting Information, Eq. S6). Although the ascertained values are similar for the two photoreactors, it is noted that the flow system was not specifically optimized for this parameter. Further improvements in the SA-to- $V$ ratio of the PCP, along with the positioning and intensity of the light source, can lead to enhancements in the EQE value.

Control experiments with the PCP in the absence of light did not result in alcohol conversion (Table 1, entry 1.ii). Using a biphasic setup (i.e. liquid-only flow, with the air feed switched off during operation), in which the substrate solution and solvent feeds were saturated with air prior to transferring into the syringes, resulted in a much lower Conv relative to the triphasic assembly (11\% compared to $80 \%$, Table 1 , entry 1.iii and 1, respectively). Purging the PCP with $\mathrm{N}_{2}$ and using $\mathrm{N}_{2}$ purged substrate and solvent feed stocks resulted in Conv of $8 \%$ (Table 1, entry 1.iv). Similarly, a low but significant Conv of $4 \%$ was measured in batch under a $\mathrm{N}_{2}$ atmosphere (Table 1, entry 1.iv). This low anaerobic background activity can be associated with the charge storage abilities of carbon nitride, whereby the oxidation and reduction steps can be decoupled, thus allowing for a small amount of substrate oxidation even in the absence of a suitable electron acceptor. ${ }^{34-36}$ Incomplete elimination of $\mathrm{O}_{2}$ within the flow setup, and the trapping of some $\mathrm{O}_{2}$ molecules within the mesoporous network of $\mathbf{m p g}-\mathbf{C N}_{\mathbf{x}},{ }^{32}$ could also contribute to this non-zero Conv value under inert conditions. These results highlight the importance of the aerobic conditions and the advantage of using a gasliquid (rather than a liquid-only) flow in which $\mathrm{O}_{2}$ acts as both an electron acceptor and a subsequent reagent in the overall oxidation process. 


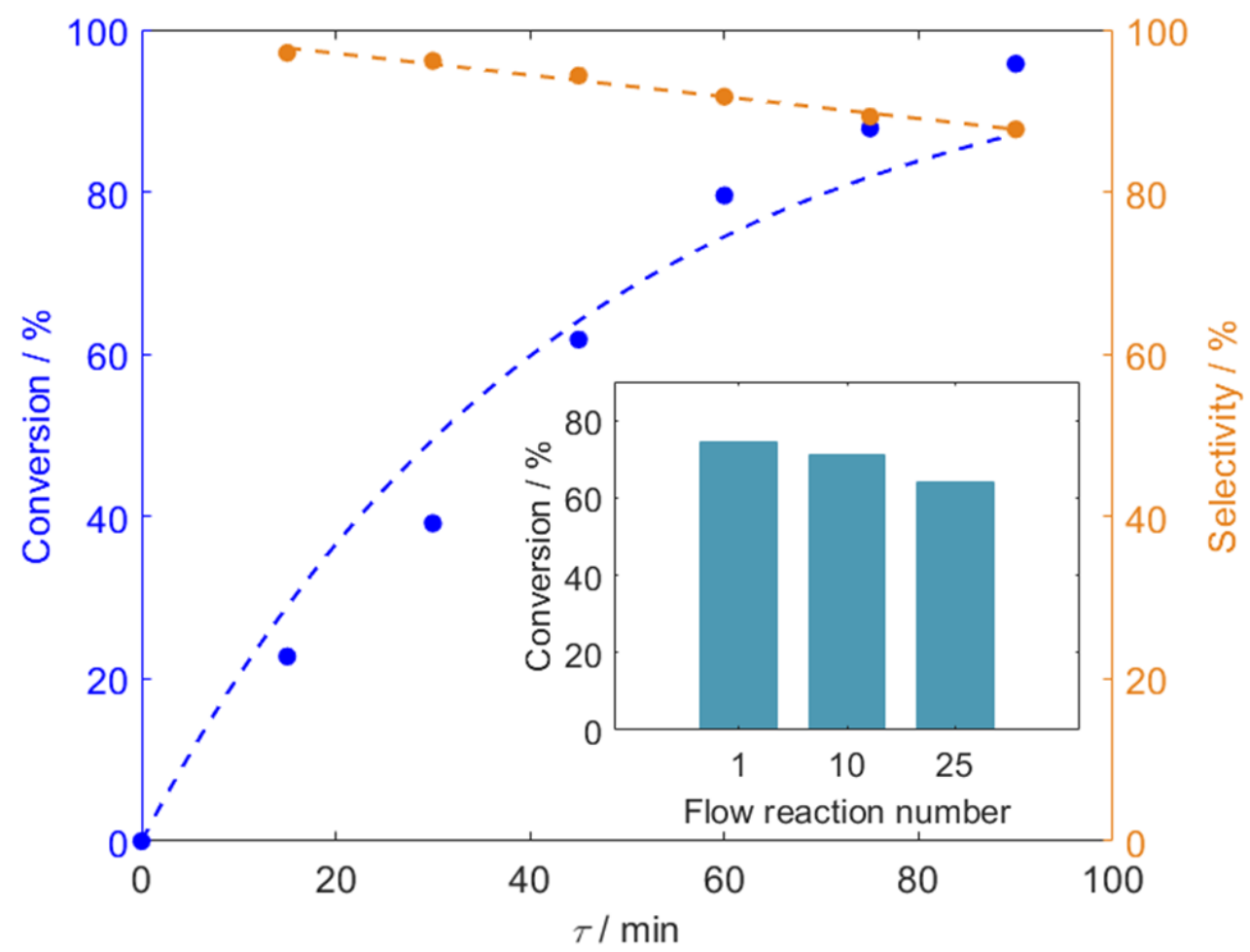

Figure 2. Residence time profile with both starting material conversion and selectivity for aldehyde production (1-naphthalenemethanol concentration $=50 \mathrm{mM}$ in MeCN). Inset: photoreactor re-usability with a total of 25 flow experiments in the PCP setup (1-naphthalenemethanol concentration $=100 \mathrm{mM}$ in MeCN, $\tau=90 \mathrm{~min})$. General conditions for both plots: $\dot{V}_{\text {air }}=0.5 \mathrm{sccm}$, blue LEDs ( $14.4 \mathrm{~W}$ at $\left.470 \mathrm{~nm}\right)$, $T=55^{\circ} \mathrm{C}$.

Para-substituents with the lowest Hammett parameter $(\sigma)$ generally achieved the highest conversions, as they are more effective in stabilizing the radical intermediate (Table 1, entries 2 and 3, versus 4 and 5; Figure S1). ${ }^{37}$ 4-tert-butylbenzyl alcohol displays a lower than expected Conv, which is presumably due to unfavorable steric effects (Table 1, entry 6). A higher Conv was achieved for all substrates under continuous flow compared to batch, as a consequence of the higher throughput efficiency provided by the PCP environment. The slightly higher Sel metric of the batch photoreactor is a consequence of the lower Conv. As discussed above (Figure 2), formation of the overoxidation product only becomes considerable once the concentration of the starting material decreases substantially, such that the oxidation of aldehyde begins to take place. Some variance in the M.B. between batch and flow was observed (average of $95 \%$ and $90 \%$ for entries 1 - 6 in Table 1, respectively; see Table S1 for full list of M.B. values), which is most likely due to the volatility of the aldehyde, which can escape from the flow reactor via the gas output stream. Thus, in 
reality, Conv and Sel for the flow PCP system are likely higher than reported in Table 1, and system improvements are currently being made to increase the $\boldsymbol{M} . \boldsymbol{B}$.

Table 1. Alcohol oxidation scope under batch and flow conditions, featuring control experiments performed in flow, based on Scheme 1a.

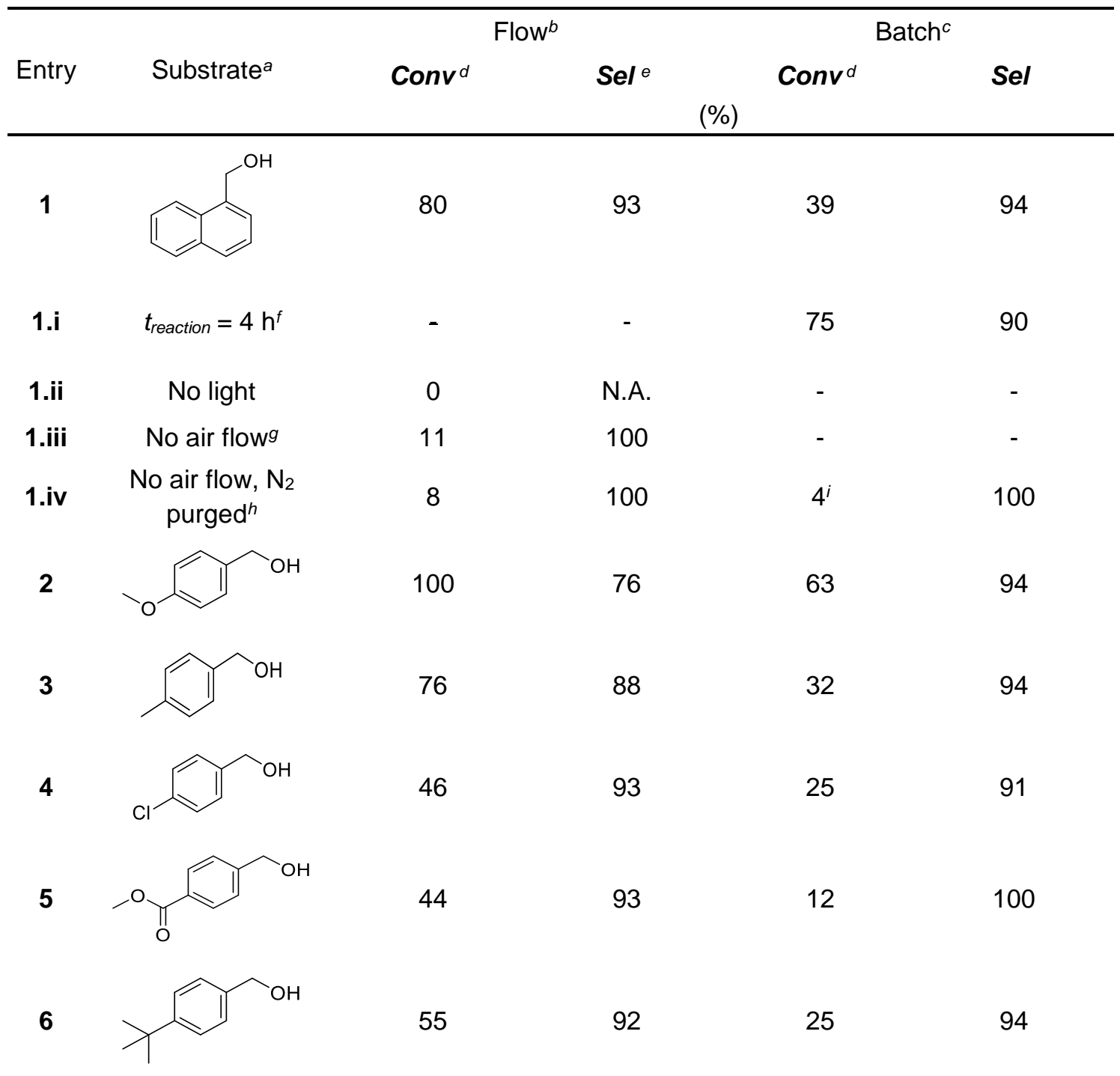

${ }^{a}$ General conditions: substrate concentration $75 \mathrm{mM}$ in $\mathrm{MeCN}\left(\mathrm{CD}_{3} \mathrm{CN}\right.$ in batch), blue LEDs (14.4 W at $470 \mathrm{~nm}$ ), $T=55^{\circ} \mathrm{C}$. Control flow or batch experiments detailed as sub-entries within the table. ${ }^{b}$ Flow conditions: $\tau=90 \mathrm{~min}, \dot{V}_{\text {air }}=0.5 \mathrm{sccm}$. ${ }^{c}$ Batch conditions: reaction time $=$ $90 \mathrm{~min}, \mathbf{m p g}-\mathrm{CN}_{\mathbf{x}}$ photocatalyst loading $=2 \mathrm{mg}$. ${ }^{d}$ Conv calculated using 1,3,5trimethoxybenzene as internal standard. ${ }^{e}$ Sel for corresponding aldehyde. ${ }^{t}$ Reaction time for batch photoreactor set to $4 \mathrm{~h}$. ${ }^{g}$ Substrate solution and solvent feeds purged with air for $15 \mathrm{~min}$ prior to transferring into the syringe. ${ }^{h}$ PCP setup first purged with $\mathrm{N}_{2}(25 \mathrm{sccm})$ for $20 \mathrm{~min}$, and then $\mathrm{MeCN}\left(1.5 \mathrm{~mL}, 0.028 \mathrm{~mL} \mathrm{~min}^{-1}\right)$; substrate solution and solvent feeds purged with $\mathrm{N}_{2}$ for 15 min prior to transferring into the syringe. ${ }^{i}$ For the batch control, a $\mathrm{N}_{2}$ atmosphere was used.

Upon replacing the alcohol by a primary benzyl amine, a dibenzylic secondary $(E)$-imine was obtained as the major product (Scheme 1b). This resulted from the cascade condensation reaction between the primary aldimine intermediate with the 
starting material in the presence of $\mathbf{m p g}-\mathbf{C N}_{\mathbf{x}}{ }^{31}$ Preliminary batch studies revealed that the oxidation of benzylic amines proceeds at a faster rate than that of benzylic alcohols - hence a lower value for $\tau$ was selected $(60 \mathrm{~min}$ ) for the continuous flow experiments. Analogous to the aerobic alcohol oxidations, the enhanced performance of the PCP in comparison to batch was observed (Table 2). We observe that the PCP setup does not impede the condensation step following initial amine oxidation. This result highlights the versatility of the flow system for various aerobic oxidation processes.

Table 2. Amine reaction scope under batch and flow conditions, featuring control experiments performed in flow, based on Scheme $1 \mathrm{~b}$.

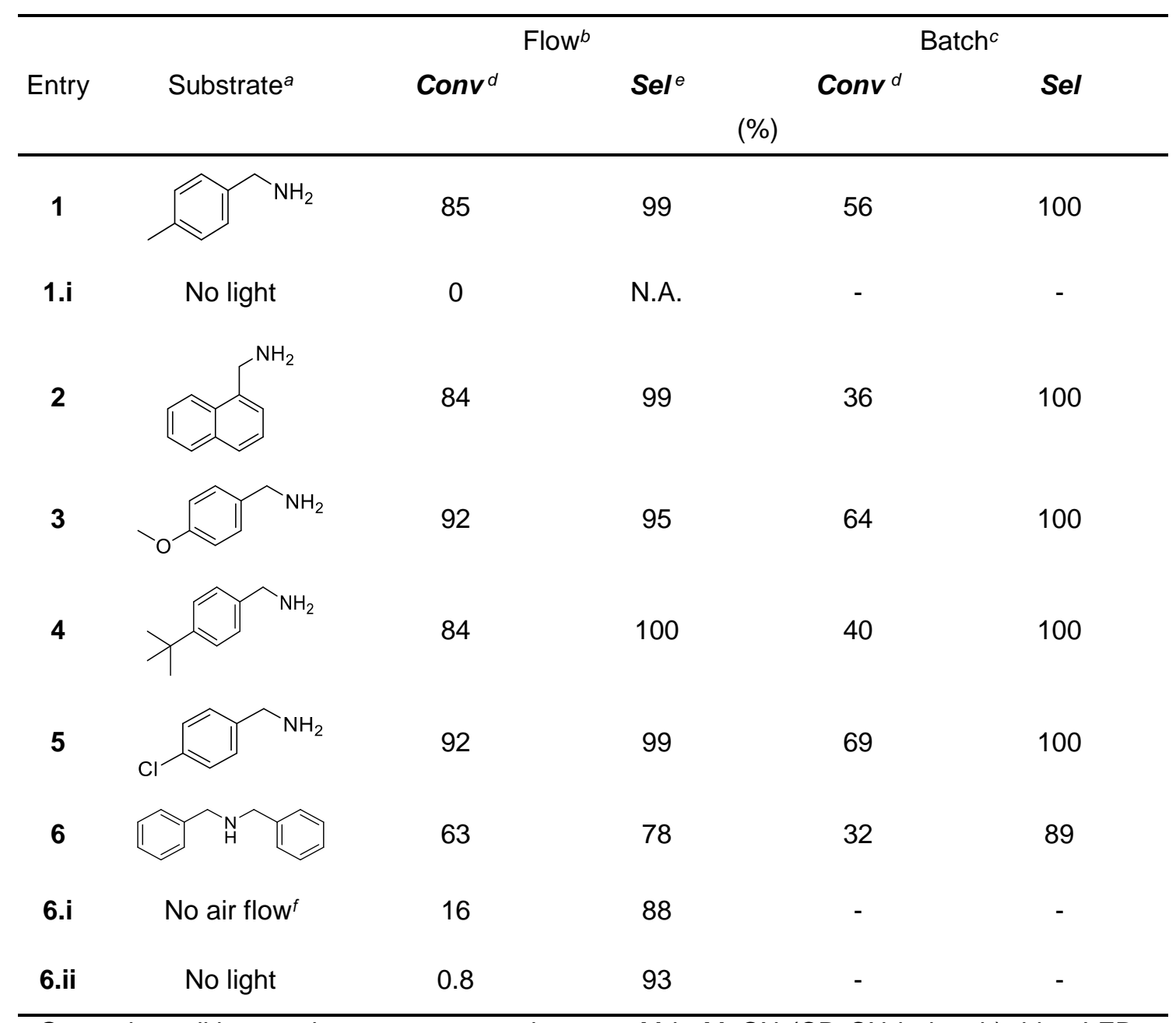

a General conditions: substrate concentration $75 \mathrm{mM}$ in $\mathrm{MeCN}\left(\mathrm{CD}_{3} \mathrm{CN}\right.$ in batch), blue LEDs $(14.4 \mathrm{~W}$ at $470 \mathrm{~nm}), T=55^{\circ} \mathrm{C}$. Control flow experiments detailed as sub-entries within the table. ${ }^{b}$ Flow conditions: $\tau=60 \mathrm{~min}, \dot{V}_{\text {air }}=0.5 \mathrm{sccm}$. ${ }^{c}$ Batch conditions: reaction time $=60 \mathrm{~min}$, mpg$\mathbf{C N}_{\mathbf{x}}$ photocatalyst loading $=2 \mathrm{mg} .{ }^{d}$ Conv calculated as per Table 1. ${ }^{e}$ Sel is for the dibenzylic secondary $(E)$-imine; minor impurity due to presence of primary benzyl imine. ${ }^{f}$ Residual $\mathrm{O}_{2}$ (from ambient air) still present in the liquid feeds. 
However, there was a discrepancy in the M.B. metric when comparing the primary amine oxidation in the PCP setup and in-batch. The M.B. for the primary amines in-flow was on average $\sim 70 \%$ (Table 2, entries 1 - 5; Table S2), whereas a M.B. of $\sim 98 \%$ was obtained in batch. Flow experiments using 1,3,5trimethoxybenzene as a tracer molecule (which was added to the amine solution and pumped simultaneously into the PCP, but was unreactive towards light activated mpg$\mathbf{C N}_{\mathbf{x}}$ ) gave complete M.B. for the tracer compound (DMSO was used as internal standard in this case). Secondary amines faced no such issue (Table 2, entry 6 ) and a $\boldsymbol{M}$. B. of $95 \%$ was achieved in-flow. This implied that a side-product from the reaction with the primary amine may have deposited within the PCP, and hence could not be accounted for during downstream liquid analysis.

High concentrations of benzylic primary amines are known to react with aerobic $\mathrm{CO}_{2}$ to form a carbamic acid/carbamate salt mixture, which consists of a white precipitate that is insoluble in a number of organic solvents. ${ }^{38,39}$ We therefore propose that the mass imbalance could originate from the formation of a carbamic acid precipitate in the PCP. To verify this hypothesis, ${ }^{13} \mathrm{CO}_{2}$ was bubbled into a primary benzyl amine solution in MeCN (100 mM). This rapidly resulted in the formation of a white precipitate within the vial, that was found to be soluble in basic solution (TBAOH, $10 \mathrm{mM}$ in MeCN). An infrared spectrum of this solid revealed the appearance of a single sharp band at $3380 \mathrm{~cm}^{-1}$, indicative of the loss of a primary amine, and characteristic peaks centered at $1650 \mathrm{~cm}^{-1}$ and $1620 \mathrm{~cm}^{-1}$, which can be attributed to the asymmetric $(\mathrm{C}=\mathrm{O})$ stretch of the $\mathrm{COOH}$ moiety of the carbamic acid and the carboxylate anion, respectively (Figure S7). ${ }^{40}$ Furthermore, a strong peak at $\sim 158 \mathrm{ppm}$ was observed for the carbamic acid carbon atom in the ${ }^{13} \mathrm{C}$ NMR spectrum (Figure S8). ${ }^{40}$ Finally, continuous flow experiments under alkaline conditions (10 mM TBAOH present in the substrate feed) increased the M.B. by $12 \%$, as the hydroxide ions facilitate hydrolysis of the carbamic acid/carbamate salt. ${ }^{41}$ Our rational understanding of the flow reaction shown in Scheme $1 \mathrm{~b}$ is that some degree of solvent evaporation occurs upon passing the solution through the heated zones $\left(\sim 55^{\circ} \mathrm{C}\right)$ of the PCP, causing the local concentration of amine to increase at such points. Here, the low pressure of $\mathrm{CO}_{2}$ from the compressed air feed is sufficient to react with the locally concentrated starting material to yield the carbamic acid/carbamate salt. Due to the insolubility of this mixture in $\mathrm{MeCN}$, it precipitates from the solution and settles in the 
PCP during operation. With the light switched off, and thus the temperature of the PCP maintained at $\sim 22{ }^{\circ} \mathrm{C}$ rather than $55{ }^{\circ} \mathrm{C}$, the M.B. for the primary benzyl amine increased from $68 \%$ to $82 \%$ (Supporting Information, Table S2, entry 1.i), supporting our rationale on the behavior of such compounds within the continuous flow environment. This kind of reaction environment is not experienced within the batch reactor, and hence, could explain the difference in the M.B. metric between the two systems. The PCP flow setup is therefore being investigated as a potential tool to explore other organic transformations, as it could potentially lead to a product distribution that differs from that obtained in a typical batch setup.

\section{Conclusions}

In conclusion, we have developed a triphasic photoreactor employing $\mathbf{m p g}-\mathbf{C N}_{\mathbf{x}}$ to explore aerobic substrate oxidations and reactions in-flow under benign conditions. We investigated the impact of the morphology of the carbon nitride within the PCP under a continuous flow environment, and established that the mesoporosity plays a vital role with regards to the gas-liquid dynamics through the heterogeneous catalyst. Our mpg- $\mathrm{CN}_{\mathbf{x}}$-based continuous flow system is not only low cost and reusable for multiple flow cycles, but is also more practical and effective than a conventional batch photoreactor. This photocatalytic flow reactor is able to conduct oxidative transformations using a well-controlled air feed, which makes it more compatible with the safety restrictions surrounding the use of $\mathrm{O}_{2}$ for industrial applications. Efforts are now being placed to utilize the overall visible light activated $\mathbf{m p g}-\mathbf{C N}_{\mathbf{x}} \mathbf{P C P}$ setup to investigate the improvement in performance of other known aerobic reactions, ${ }^{31,42,43}$ and to probe novel organic transformations under continuous flow operation.

\section{Associated Content}

Further details on the experimental conditions $\left(\mathbf{m p g}-\mathbf{C N}_{\mathbf{x}}\right.$ fabrication, batch and flow photoreactor setup, MeCN suppression ${ }^{1} \mathrm{H}$ NMR programme), extra figures (TEM, nitrogen sorption data, ${ }^{13} \mathrm{C}$ NMR and FTIR spectra, optimization plots, images of the flow assembly), and extra tables (list of mass balance values) can be found in the Supporting Information. 


\section{Conflict of interest}

There are no conflicts of interest to declare.

\section{Acknowledgements}

This work was supported by an Endeavour Scholarship (M.B.), the Cambridge Trust (A.V.), the Christian Doppler Research Association (Austrian Federal Ministry for Digital and Economic Affairs and the National Foundation for Research, Technology and Development), and OMV (M.B., E.R.). We thank Prof. Yubao Zhao for initial $\mathbf{m p g}-\mathbf{C N}_{\mathbf{x}}$ preparation, Dr. Tobias Heil for TEM measurements, Dr. Ricardo Labes, Dr. Fabio Lima, and Prof. Steven V. Ley for their great advice with regards to flow photochemistry, and Prof. Markus Antonietti and Dr. Julien Warnan for helpful discussions.

\section{References}

(1) Pastre, J. C.; Browne, D. L.; Ley, S. V. Flow Chemistry Syntheses of Natural Products. Chem. Soc. Rev. 2013, 42, 8849-8869.

(2) Su, Y.; Straathof, N. J. W.; Hessel, V.; Noël, T. Photochemical Transformations Accelerated in Continuous-Flow Reactors: Basic Concepts and Applications. Chem. Eur. J. 2014, 20, 10562-10589.

(3) Cambié, D.; Bottecchia, C.; Straathof, N. J. W.; Hessel, V.; Noël, T. Applications of Continuous-Flow Photochemistry in Organic Synthesis, Material Science, and Water Treatment. Chem. Rev. 2016, 116, 10276-10341.

(4) Mizuno, K.; Nishiyama, Y.; Ogaki, T.; Terao, K.; Ikeda, H.; Kakiuchi, K. Utilization of Microflow Reactors to Carry out Synthetically Useful Organic Photochemical Reactions. J. Photochem. Photobiol. C Photochem. Rev. 2016, 29, 107-147.

(5) Plutschack, M. B.; Pieber, B.; Gilmore, K.; Seeberger, P. H. The Hitchhiker's Guide to Flow Chemistry. Chem. Rev. 2017, 117, 11796-11893.

(6) Loubière, K.; Oelgemöller, M.; Aillet, T.; Dechy-Cabaret, O.; Prat, L. ContinuousFlow Photochemistry: A Need for Chemical Engineering. Chem. Eng. Process. 2016, 104, 120-132.

(7) Newman, S. G.; Jensen, K. F. The Role of Flow in Green Chemistry and Engineering. Green Chem. 2013, 15, 1456-1472. 
(8) Gutmann, B.; Cantillo, D.; Kappe, C. O. Continuous-Flow Technology-A Tool for the Safe Manufacturing of Active Pharmaceutical Ingredients. Angew. Chem. Int. Ed. 2015, 54, 6688-6728.

(9) Douglas, J. J.; Sevrin, M. J.; Stephenson, C. R. J. Visible Light Photocatalysis: Applications and New Disconnections in the Synthesis of Pharmaceutical Agents. Org. Process Res. Dev. 2016, 20, 1134-1147.

(10) Knowles, J. P.; Elliott, L. D.; Booker-Milburn, K. I. Flow Photochemistry: Old Light through New Windows. Beilstein J. Org. Chem. 2012, 8, 2025-2052.

(11) Shaw, M. H.; Twilton, J.; MacMillan, D. W. C. Photoredox Catalysis in Organic Chemistry. J. Org. Chem. 2016, 81, 6898-6926.

(12) Romero, N. A.; Nicewicz, D. A. Organic Photoredox Catalysis. Chem. Rev. 2016, 116, 10075-10166.

(13) Wang, Y.; Wang, X.; Antonietti, M. Polymeric Graphitic Carbon Nitride as a Heterogeneous Organocatalyst: From Photochemistry to Multipurpose Catalysis to Sustainable Chemistry. Angew. Chem. Int. Ed. 2012, 51, 68-89.

(14) Savateev, A.; Ghosh, I.; König, B.; Antonietti, M. Photoredox Catalytic Organic Transformations Using Heterogeneous Carbon Nitrides. Angew. Chem. Int. Ed. 2018, 57, 15936-15947.

(15) Vijeta, A.; Reisner, E. Carbon Nitride as a Heterogeneous Visible-Light Photocatalyst for the Minisci Reaction and Coupling to $\mathrm{H}_{2}$ Production. Chem. Commun. 2019, 55, 14007-14010.

(16) Woźnica, M.; Chaoui, N.; Taabache, S.; Blechert, S. THF: An Efficient Electron Donor in Continuous Flow Radical Cyclization Photocatalyzed by Graphitic Carbon Nitride. Chem. Eur. J. 2014, 20, 14624-14628.

(17) Pieber, B.; Shalom, M.; Antonietti, M.; Seeberger, P. H.; Gilmore, K. Continuous Heterogeneous Photocatalysis in Serial Micro-Batch Reactors. Angew. Chem. Int. Ed. 2018, 57, 9976-9979.

(18) Guo, Z.; Liu, B.; Zhang, Q.; Deng, W.; Wang, Y.; Yang, Y. Recent Advances in Heterogeneous Selective Oxidation Catalysis for Sustainable Chemistry. Chem. Soc. Rev. 2014, 43, 3480.

(19) Gavriilidis, A.; Constantinou, A.; Hellgardt, K.; Hii, K. K. (Mimi); Hutchings, G. J.; Brett, G. L.; Kuhn, S.; Marsden, S. P. Aerobic Oxidations in Flow: Opportunities for the Fine Chemicals and Pharmaceuticals Industries. React. Chem. Eng. 2016, 1, 595-612. 
(20) Caron, S.; Dugger, R. W.; Ruggeri, S. G.; Ragan, J. A.; Brown Ripin, D. H. Large-Scale Oxidations in the Pharmaceutical Industry. Chem. Rev. 2006, 106, 2943-2989.

(21) Marui, K.; Nomoto, A.; Akashi, H.; Ogawa, A. Green Oxidation of Amines to Imines Based on the Development of Novel Catalytic Systems Using Molecular Oxygen or Hydrogen Peroxide. Synth. 2016, 48, 31-42.

(22) Wu, G.; Cao, E.; Ellis, P.; Constantinou, A.; Kuhn, S.; Gavriilidis, A. Development of a Flat Membrane Microchannel Packed-Bed Reactor for Scalable Aerobic Oxidation of Benzyl Alcohol in Flow. Chem. Eng. J. 2018, 19.

(23) Emmanuel, N.; Mendoza, C.; Winter, M.; Horn, C. R.; Vizza, A.; Dreesen, L.; Heinrichs, B.; Monbaliu, J. M. Scalable Photocatalytic Oxidation of Methionine under Continuous-Flow Conditions. Org. Process Res. Dev. 2017, 21, 14351438.

(24) Liu, K.; Jiang, S.; Lu, L.; Tang, L.-L.; Tang, S.-S.; Tang, H.-S.; Tang, Z.; He, W.; Xu, X. Bis(Methoxypropyl) Ether-Promoted Oxidation of Aromatic Alcohols into Aromatic Carboxylic Acids and Aromatic Ketones with $\mathrm{O} 2$ under Metal- and Base-Free Conditions. Green Chem. 2018, 20, 3038-3043.

(25) Schilling, W.; Riemer, D.; Zhang, Y.; Hatami, N.; Das, S. Metal-Free Catalyst for Visible-Light-Induced Oxidation of Unactivated Alcohols Using Air/Oxygen as an Oxidant. ACS Catal. 2018, 8, 5425-5430.

(26) Frost, C. G.; Mutton, L. Heterogeneous Catalytic Synthesis Using Microreactor Technology. Green Chem. 2010, 12, 1687-1703.

(27) Munirathinam, R.; Huskens, J.; Verboom, W. Supported Catalysis in Continuous-Flow Microreactors. Adv. Synth. Catal. 2015, 357, 1093-1123.

(28) Irfan, M.; Glasnov, T. N.; Kappe, C. O. Heterogeneous Catalytic Hydrogenation Reactions in Continuous-Flow Reactors. ChemSusChem 2011, 4, 300-316.

(29) Ricciardi, R.; Huskens, J.; Verboom, W. Nanocatalysis in Flow. ChemSusChem 2015, 8, 2586-2605.

(30) Su, F.; Mathew, S. C.; Lipner, G.; Fu, X.; Antonietti, M.; Blechert, S.; Wang, X. Mpg- $\mathrm{C}_{3} \mathrm{~N}_{4}$-Catalyzed Selective Oxidation of Alcohols Using $\mathrm{O}_{2}$ and Visible Light. J. Am. Chem. Soc. 2010, 132, 16299-16301.

(31) Su, F.; Mathew, S. C.; Möhlmann, L.; Antonietti, M.; Wang, X.; Blechert, S. Aerobic Oxidative Coupling of Amines by Carbon Nitride Photocatalysis with 
Visible Light. Angew. Chem. Int. Ed. 2011, 50, 657-660.

(32) Li, X. H.; Wang, X.; Antonietti, M. Solvent-Free and Metal-Free Oxidation of Toluene Using $\mathrm{O}_{2}$ and $\mathrm{g}-\mathrm{C}_{3} \mathrm{~N}_{4}$ with Nanopores: Nanostructure Boosts the Catalytic Selectivity. ACS Catal. 2012, 2, 2082-2086.

(33) Coyle, E. E.; Oelgemöller, M. Micro-Photochemistry: Photochemistry in Microstructured Reactors. The New Photochemistry of the Future? Photochem. Photobiol. Sci. 2008, 7, 1313.

(34) Kasap, H.; Caputo, C. A.; Martindale, B. C. M.; Godin, R.; Lau, V. W.-H.; Lotsch, B. V.; Durrant, J. R.; Reisner, E. Solar-Driven Reduction of Aqueous Protons Coupled to Selective Alcohol Oxidation with a Carbon Nitride-Molecular $\mathrm{Ni}$ Catalyst System. J. Am. Chem. Soc. 2016, 138, 9183-9192.

(35) Lau, V. W.; Klose, D.; Kasap, H.; Podjaski, F.; Pignié, M.-C.; Reisner, E.; Jeschke, G.; Lotsch, B. V. Dark Photocatalysis: Storage of Solar Energy in Carbon Nitride for Time-Delayed Hydrogen Generation. Angew. Chem. Int. Ed. 2017, 56, 510-514.

(36) Savateev, A.; Kurpil, B.; Mishchenko, A.; Zhang, G.; Antonietti, M. A "Waiting" Carbon Nitride Radical Anion: A Charge Storage Material and Key Intermediate in Direct C-H Thiolation of Methylarenes Using Elemental Sulfur as the "S"Source. Chem. Sci. 2018, 9, 3584-3591.

(37) McDaniel, D. H.; Brown, H. C. An Extended Table of Hammett Substituent Constants Based on the lonization of Substituted Benzoic Acids. J. Org. Chem. 1958, 23, 420-427.

(38) Patchornik, A.; Shalitin, Y. Titrimetric Method for Continuous Determination of Carbon Dioxide and Its Application in Amino Acid Chemistry. Anal. Chem. 1961, 33, 1887-1889.

(39) Richner, G.; Puxty, G.; Carnal, A.; Conway, W.; Maeder, M.; Pearson, P. Thermokinetic Properties and Performance Evaluation of Benzylamine-Based Solvents for CO2 Capture. Chem. Eng. J. 2015, 264, 230-240.

(40) Liu, A. H.; Ma, R.; Song, C.; Yang, Z. Z.; Yu, A.; Cai, Y.; He, L. N.; Zhao, Y. N.; Yu, B.; Song, Q. W. Equimolar CO 2 Capture by N-Substituted Amino Acid Salts and Subsequent Conversion. Angew. Chem. Int. Ed. 2012, 51, 11306-11310.

(41) Dittert, L. W.; Higuchi, T. Rates of Hydrolysis of Carbamate and Carbonate Esters in Alkaline Solution. J. Pharm. Sci. 1963, 52, 852-857.

(42) Song, T.; Zhou, B.; Peng, G.-W.; Zhang, Q.-B.; Wu, L.-Z.; Liu, Q.; Wang, Y. 
Aerobic Oxidative Coupling of Resveratrol and Its Analogues by Visible Light Using Mesoporous Graphitic Carbon Nitride (Mpg-C 3 N 4 ) as a Bioinspired Catalyst. Chem. Eur. J. 2014, 20, 678-682.

(43) Zhao, Y.; Antonietti, M. Visible-Light-Irradiated Graphitic Carbon Nitride Photocatalyzed Diels-Alder Reactions with Dioxygen as Sustainable Mediator for Photoinduced Electrons. Angew. Chem. Int. Ed. 2017, 56, 9336-9340. 
TOC

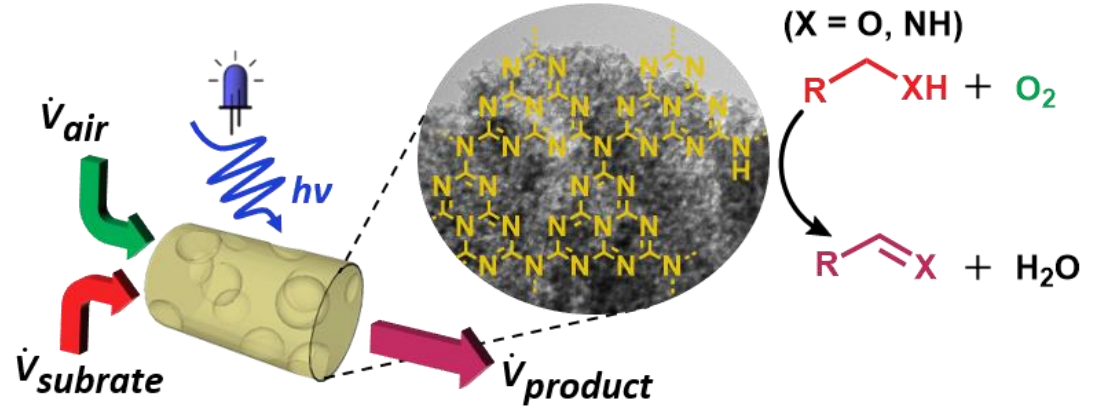

